\title{
Levitation mechanism of impacting nanodroplet on heated wall
}

\author{
Hirofumi Tabe ${ }^{\mathrm{a}, *}$, Kazumichi Kobayashi ${ }^{\mathrm{a}}$, Hisao Yaguchi ${ }^{\mathrm{b}}$, Hiroyuki Fujii ${ }^{\mathrm{a}}$, Masao \\ Watanabe \\ ${ }^{a}$ Division of Mechanical and Space Engineering, Hokkaido University, Sapporo, 060-8628 Japan \\ ${ }^{b}$ Department of Mechanical Engineering, National Institute of Technology, Gunma College, Maebashi 371-8530, Japan
}

\begin{abstract}
Levitation of a droplet after impacting on a high-temperature wall is well known as the Leidenfrost effect. In this study, we conducted a molecular dynamics (MD) simulation to elucidate the mechanism of nanodroplet levitation and to investigate the relation between the levitation mechanism and liquid-solid intermolecular force. We found that the impacting nanodroplet levitated from its edge on the heated wall. Analyses on velocity fields inside the droplet indicated that an upward velocity at the edge of the nanodroplet was caused by an internal flow. The high wall temperature or strong liquid-solid intermolecular force caused an intensive evaporation near the three-phase contact line before levitation occurred, and this intensive evaporation yielded a large internal flow within the nanodroplet.
\end{abstract}

Keywords: Molecular dynamics simulation, Leidenfrost effect, Intermolecular force, Three-phase contact line, Internal flow

\section{Introduction}

A liquid droplet can levitate after impacting on a high-temperature wall [1]. A vapor film is generated by an instantaneous evaporation in the vicinity of the wall and the droplet levitates owing to this vapor film. This levitation phenomenon is widely known as the Leidenfrost effect [2], and a large amount of studies have been conducted to investigate this phenomenon.

A primary research scope of this phenomenon is the Leidenfrost temperature that is defined as the minimum temperature of the heated wall at which the Leidenfrost effect occurs. It is known that several parameters affect the Leidenfrost temperature such as the Weber number of the droplet, ambient gas pressure, and wettability of the solid wall. Some studies have reported that a droplet of high Weber number increases the Leidenfrost temperature because of the balance between the inertial pressure of the droplet and the induced vapor pressure [3, 4]. Further, it has been demonstrated that the Leidenfrost temperature decreased with decreasing ambient gas pressure [5, 6]. Almost consistent results were obtained for these factors.

Investigations with respect to the influence of wettability on the Leidenfrost temperature have been conducted actively. Wettability varies depending on the interaction between liquid and solid or the surface structures of the solid wall. It has been reported that the Leidenfrost temperature had increased on hydrophilic surfaces yielded by plasma irradiation [7], while it decreased on hydrophobic surfaces yielded by Teflon or silane coating $[8,9]$. In addition, some studies indicated that structured surfaces decreased

${ }^{*}$ Corresponding author.

Email address: azx@eis .hokudai .ac.jp (Hirofumi Tabe) 
the Leidenfrost temperature $[9,10,11]$, whereas structured surfaces increased the Leidenfrost temperature $[12,13]$.

Although many studies regarding the Leidenfrost effect have been conducted, a detailed mechanism of this phenomenon and the influence of wettability on the Leidenfrost effect are still not clarified. Therefore, in this study, we conducted molecular dynamics (MD) simulations to elucidate the mechanism of the Leidenfrost effect by investigating the internal flow and temperature fields of impacting droplet. Because the MD simulation is a molecular-scale analysis method, we can investigate phase-change phenomena without any special condition. Studies related to the evaporation of a sessile droplet or liquid film $[14,15,16,17,18]$, bubble nucleation [19,20,21], or condensation [22] in multiphase systems composed of liquid and solid molecules have been conducted using MD simulations. Although the obtained results are mostly considered as the nanoscale phenomena, MD simulations are suitable for the detailed analysis of the Leidenfrost effect, which is the instantaneous phenomenon with phase change.

In addition, we can easily adjust the magnitude of the intermolecular force in MD simulations. An interaction between liquid and solid molecules is relevant to the wettability of solid surfaces. Typically, strong liquid-solid intermolecular forces result in hydrophilic walls whereas weak forces result in hydrophobic walls. It was reported that same tendency was reproduced in nanoscale MD simulations [16, 23, 24]. In our previous MD study [25], we found that a strong liquid-solid intermolecular force decreases the Leidenfrost temperature. However, the connection has not been established between the influence of the liquid-solid intermolecular force and the mechanism of the Leidenfrost effect. Thus, after clarifying the levitation mechanism, we also conducted an investigation of the influence of the liquid-solid intermolecular force on this mechanism.

\section{Method}

Our calculation domain consisted of an argon nanodroplet, argon vapor, and a platinum wall. The number of utilized molecules were 26,770 for argon and 140,400 for platinum. The dimensions of the calculation domain were $43.3 \mathrm{~nm}, 43.2 \mathrm{~nm}$, and $40.0 \mathrm{~nm}$ in the $x$-, $y$-, and $z$-directions, respectively. Periodic boundary conditions were imposed in $x$ - and $y$-directions. In the $z$-direction, a mirror boundary condition was imposed on the top surface of the calculation domain. A platinum wall consisted of three layers of platinum molecules, in which they were arranged to reproduce a fcc(111) surface. To control the wall temperature using the Langevin method [26], two control layers were inserted below the three layers. The initial temperature of the wall was set to $T_{w}=85 \mathrm{~K}$.

The argon droplet and its vapor were equilibrated at $T_{0}=85 \mathrm{~K}$ using the velocity scaling method [27]. After reaching the equilibrium state, the platinum wall was inserted to the bottom of the calculation domain and the initial condition of simulations was formed. The initial diameter of the nanodroplet was $12.60 \mathrm{~nm}$. After establishing the initial condition, the droplet molecules were assigned an initial velocity toward the platinum wall. The initial velocity $V_{0}$ was $-100 \mathrm{~m} / \mathrm{s}$ and the corresponding Weber number was $W e=13.6$. For calculating $W e$, we applied $\rho=1400 \mathrm{~kg} / \mathrm{m}^{3}$ for the density and $\gamma=0.013 \mathrm{~N} / \mathrm{m}$ for the surface tension, which were derived from the previous nanoscale MD study [27]. Although the initial velocity of the droplet was too high, the order of the Weber number agreed with that of previous experimental studies $[3,4,11,13]$. When the droplet molecules were assigned the initial velocity, the wall temperature began to increase. It took approximately $140 \mathrm{ps}$ for the droplet to impact, whereas it took approximately $50 \mathrm{ps}$ for wall temperature to reach the target temperature. Therefore, there was enough time until the wall temperature increased sufficiently. In the Langevin method, the wall was set to maintain the desired temperature through a simulation.

For the intermolecular potential between argon molecules, we used the following 12-6 Lennard-Jones potential function:

$$
\phi_{\mathrm{Ar}}(r)=4 \varepsilon_{\mathrm{Ar}}\left[\left(\frac{\sigma_{\mathrm{Ar}}}{r}\right)^{12}-\left(\frac{\sigma_{\mathrm{Ar}}}{r}\right)^{6}\right]
$$

where the potential depth $\varepsilon_{\mathrm{Ar}}$ is $1.635 \times 10^{-21} \mathrm{~J}$, the molecular radius $\sigma_{\mathrm{Ar}}$ is $0.3405 \mathrm{~nm}$, and $r$ is the distance between molecules. For the interaction between platinum molecules, we used the following harmonic 
oscillator function:

$$
\phi_{\mathrm{Pt}}(r)=\frac{1}{2} k\left(r-r_{0}\right)^{2}
$$

where the spring constant $k$ is $46.8 \mathrm{~N} / \mathrm{m}$ and the lattice constant of platinum $r_{0}$ is $0.2774 \mathrm{~nm}$. The platinum wall in this study is just an approximated model with the lattice vibrations. However, it is much simpler than the complex interactions of the real platinum. Owing to its effectiveness, it is widely used to investigate the heat transfer between solid and liquid in many MD simulations.

For the intermolecular potential between the argon and platinum molecules, the following potential parameters are derived from the Lorentz-Berthelot mixing rules:

$$
\sigma_{\mathrm{int}}=\frac{\sigma_{\mathrm{Ar}}+\sigma_{\mathrm{Pt}}}{2}, \varepsilon_{\mathrm{int}}=\sqrt{\varepsilon_{\mathrm{Ar}} \cdot \varepsilon_{\mathrm{Pt}}}
$$

where $\varepsilon_{\mathrm{Pt}}=8.346 \times 10^{-20} \mathrm{~J}$ and $\sigma_{\mathrm{Pt}}=0.2475 \mathrm{~nm}$. However, it is known that this Lorentz-Berthelot mixing rules yield overly attractive parameters [28]. To resolve this problem, we used the following potential function and potential parameters [29, 30]:

$$
\phi_{\mathrm{Ar}-\mathrm{Pt}}(r)=4 \varepsilon_{\mathrm{Ar}-\mathrm{Pt}}\left[\left(\frac{\sigma_{\mathrm{Ar}-\mathrm{Pt}}}{r}\right)^{12}-\beta\left(\frac{\sigma_{\mathrm{Ar}-\mathrm{Pt}}}{r}\right)^{6}\right]
$$

with

$$
\sigma_{\mathrm{Ar}-\mathrm{Pt}}=\frac{\sigma_{\mathrm{Ar}}+\sigma_{\mathrm{Pt}}}{2}, \varepsilon_{\mathrm{Ar}-\mathrm{Pt}}=\alpha \sqrt{\varepsilon_{\mathrm{Ar}} \cdot \varepsilon_{\mathrm{Pt}}} .
$$

We can adjust the strength of the intermolecular force between argon and platinum molecules by varying the values of $\alpha$ and $\beta$ in Eqs. 4 and 5. Sedighi et al. [23] varied the liquid-solid intermolecular force in their MD simulations and compared the behavior of an impacting droplet on an unheated wall with a previous experimental study [31]. They reported that similar behaviors shown in experiments could be reproduced in MD simulations on several wettabilities by adjusting the liquid-solid intermolecular force. In Section 3.6, we varied the strength of the intermolecular force to investigate the relation with the levitation mechanism. Based on the values in Sedighi's study, we varied $\alpha=0.07,0.14$, and 1.0 with $\beta=1.0$. The solid walls indicated hydrophilicity for $\alpha=0.07$ and strong hydrophilicity for $\alpha=1.0$.

When the value of $\alpha$ became high, the vapor molecules adsorbed to the platinum wall before the droplet impact owing to the strong intermolecular force even if the wall temperature was high. Because of the difference in saturated vapor pressure between the liquid droplet and thin adsorbed film, it was impossible to establish the adsorbed film into the initial condition beforehand with aligning the number of argon molecules and the initial diameter of the droplet. To maintain the condition of the solid surface at the time of impacting for all simulations, we set $\alpha=0.007$ for the vapor-solid intermolecular force. The same setting had also been used in our previous MD study [25] to avoid the initial adsorption of vapor molecules.

The cutoff radius was set as $r_{c}=1.5 \mathrm{~nm}$ and the time step for calculation was $\Delta t=5 \mathrm{fs}$. Newton's equations of motion for each molecule were solved using the leap-flog method. Because we considered the droplet impact as an axisymmetric phenomenon, we set the double-cylinder control volumes [25, 32], as shown in Fig. 1. These control volumes consisted of 63 layers and 63 shells. Their height $d z$ and thickness $d r$ were $d z=d r=\sigma_{\mathrm{Ar}}$. We calculated the density, velocity, and temperature fields using these control volumes to investigate the details of the Leidenfrost effect. The density fields were derived from the number of argon molecules in each cell of the control volumes; similarly, the velocity fields were calculated from the velocities of argon molecules in each cell. Furthermore, the temperature can be calculated only from the translation energy because the argon is a monatomic molecule. Thus, the temperature fields were also derived from the velocities of argon molecules. Detailed method to calculate the temperature fields is described in Section 3.3. We calculated the density, velocity, and temperature fields every 2 ps by averaging these values in each cell of the control volumes for 400 steps. Part of the calculated density field and the corresponding simulation domain are shown in Fig. 2. Layer 1 and Shell 1 were defined as the bottom layer and innermost shell of the control volumes, respectively. Shell 1 had the smallest volume of these control volumes. However, since its radius and height were $\sigma_{\mathrm{Ar}}$ and there was at least one molecule in it, the strong 


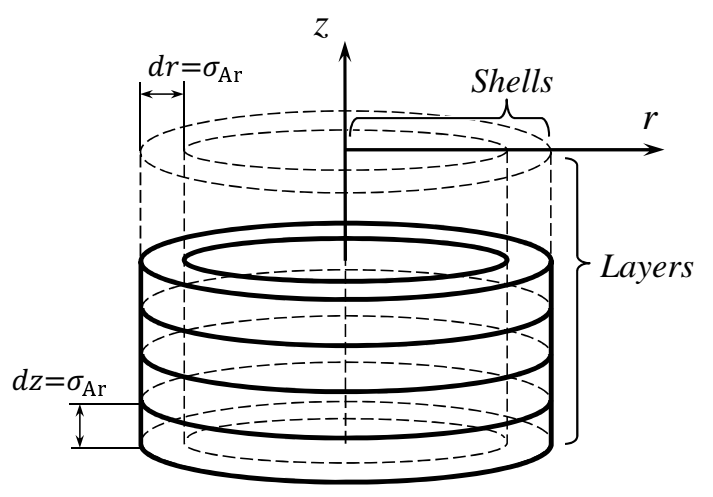

Fig. 1. Control volumes of this MD simulation. There are 63 layers and 63 shells.

density fluctuation did not occur in Shell 1 of the control volumes. The cross points in the right part of Fig. 2 represent the center of each cell of the control volumes, and the densities calculated in each cell were corresponded to the cross points of Fig. 2. Thus, the squares in Fig. 2 do not represent the cells of the control volumes.

The liquid and vapor phases must be distinguished clearly to define the exact time of the occurrence of the levitation or the evaporation amount from the liquid droplet. Therefore, to binarize density fields, we set the threshold density as $430 \mathrm{~kg} / \mathrm{m}^{3}$, which is derived from the critical density of the Lennard-Jones fluid with the potensial pareameters of argon [33]: $\rho_{\mathrm{c}}=430.26 \mathrm{~kg} / \mathrm{m}^{3}$. Kobayashi et al. [32] reported that the molecules presented above Layer 4 were hardly affected by the intermolecular force with the solid wall owing to the balance between the cutoff radius and height of the layers of the control volumes. Hence, we defined the case where the liquid phase levitated above Layer 4 as the occurrence of the Leidenfrost effect in this MD study. According to this definition, the moment of the occurrence of the Leidenfrost effect was determined from the time when the liquid phase levitated above Layer 4 and there is no liquid phase in Layer 4.

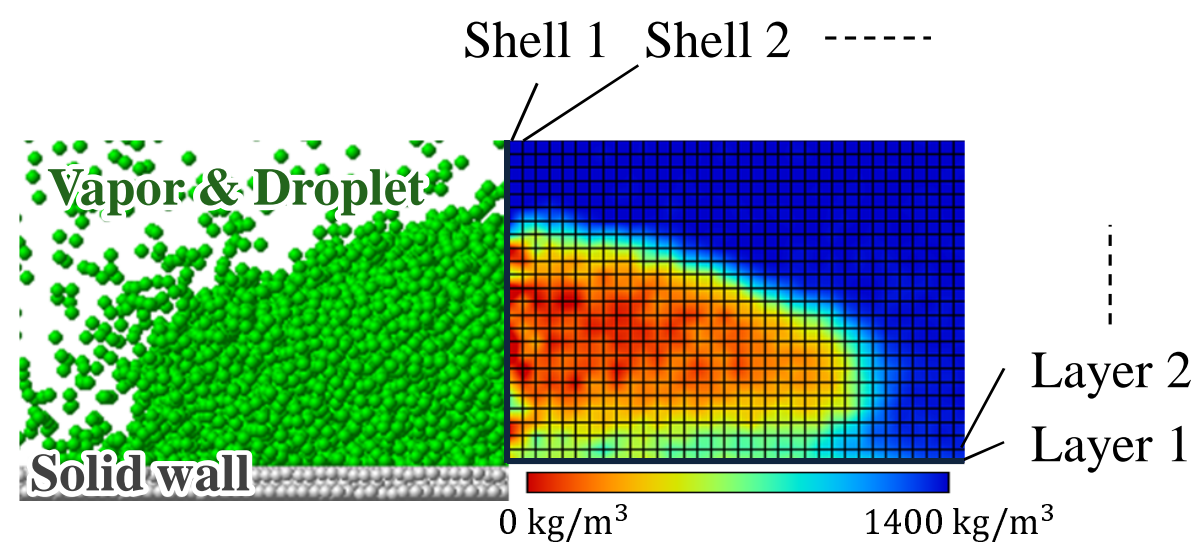

Fig. 2. Parts of simulation domain (left side) and calculated density field (right side). Layer 1 and shell 1 are set at bottom and innermost of control volumes, respectively.

Further, since the molecular mass is quite small, we did not impose the gravitational acceleration on all molecules in the present MD simulations. We have conducted several simulations with imposing the gravitational acceleration on the argon molecules, and have confirmed that the results do not change with or without the gravitational acceleration. Once the Leidenfrost effect occurred, the droplet did not stay near the heated wall and continued to rise in this study; this was not related to the setting without imposing the 
gravitational accelaration.

\section{Results}

In this section, we first discuss the levitation mechanism of the nanodroplet for $\alpha=0.14$ as a representative. Subsequently, we discuss the influence of the liquid-solid intermolecular force on the levitation mechanism.

\subsection{Behavior of impacting droplet on heated wall}

Figure 3 shows the binary density images of the impacting droplet on the heated wall for $\alpha=0.14$. The droplet impacted on the wall at $t=0 \mathrm{ps}$. The last figures in Figs. $3 \mathrm{~b}$ and $3 \mathrm{c}$ are the droplet shapes at the time when the Leidenfrost effect occurred. As shown in Fig. 3a, the impacting droplet evaporated and did not levitate on the wall at $T_{w}=213 \mathrm{~K}$. Meanwhile, as shown in Figs. 3b and 3c, the droplet levitated after impacting on the wall at $T_{w}=255 \mathrm{~K}$ or $340 \mathrm{~K}$. In addition, the Leidenfrost effect occurred earlier when $T_{w}=340 \mathrm{~K}$ than when $T_{w}=255 \mathrm{~K}$. The Leidenfrost effect is more likely to occur at a higher wall temperature.

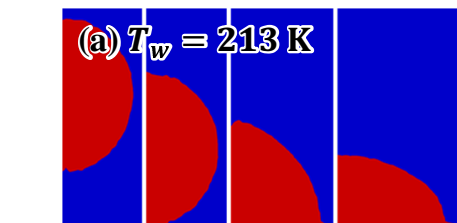

$t=-50 \mathrm{ps} \quad 0 \mathrm{ps}$

$50 \mathrm{ps}$

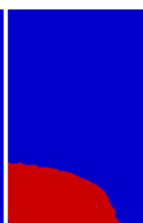

150 ps

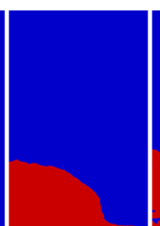

250 ps

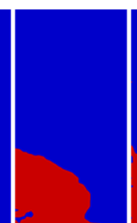

350 ps

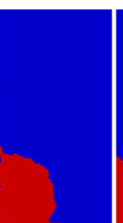

450 ps 550 ps
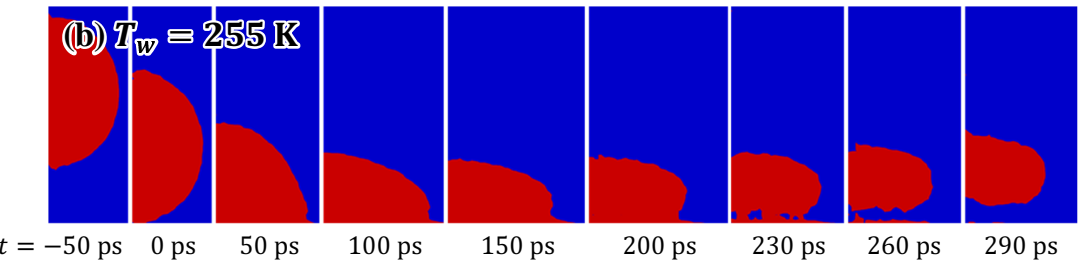

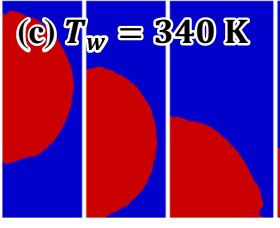

$t=-50 \mathrm{ps} \quad 0 \mathrm{ps}$

$50 \mathrm{ps}$

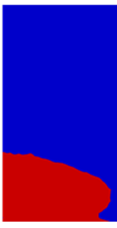

100 ps

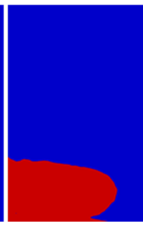

120 ps

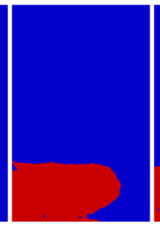

140 ps

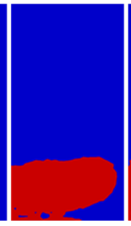

160 ps $\quad 180$ ps $\quad 218$ ps

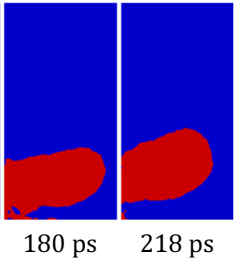

Fig. 3. Binarized density field of impacting droplet on heated wall for $\alpha=0.14$ at (a) $T_{w}=213 \mathrm{~K}$, (b) $T_{w}=255 \mathrm{~K}$, and (c) $T_{w}=340 \mathrm{~K}$.

Additionally, Fig. 3 shows that a thin argon film is adsorbed on the heated wall for all cases. It was reported that this adsorption layer was formed owing to a strong liquid-solid intermolecular force, and the temperature of the adsorption layer was almost same as that of a heated wall [25]. For $\alpha=0.14$, an adsorption layer was formed in Layer 1 of the control volumes when the droplet impacted on the heated wall. After droplet levitation, the adsorption layer evaporated gradually and disappeared, as shown in Figs. $3 \mathrm{~b}$ and $3 \mathrm{c}$. It was reported that an adsorbed region where evaporation does not occur is present owing to strong intermolecular forces in the vicinity of the contact line of the liquids on a solid wall at the microscopic scale in reality $[34,35]$. We assumed that this non-evaporating region corresponds to the adsorption layer in Layer 1 under the condition of the present MD study. The droplets levitated from above the adsorption layer, and were separated primarily from Layers 2-4, as shown in Fig. 4.

The shapes of the levitating droplet are obviously different as shown in Figs. $3 \mathrm{~b}$ and $3 \mathrm{c}$. The droplet levitates almost horizontally on the wall at $T_{w}=255 \mathrm{~K}$; meanwhile, the droplet levitates from its edge 


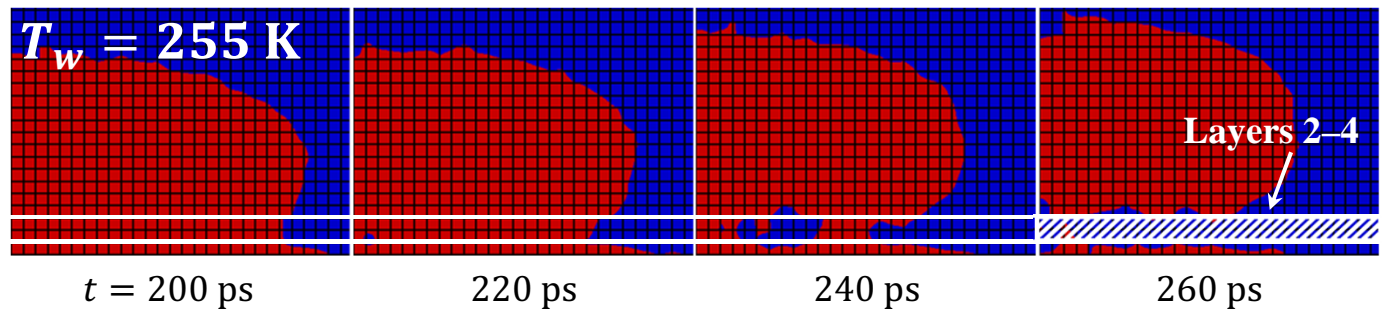

Fig. 4. Evaporating and separating position of droplet.

and tilts at $T_{w}=340 \mathrm{~K}$. A higher upward velocity may occur at the edge of the droplet when the wall temperature is higher. Therefore, we focus on the velocities caused in the levitating droplet next.

\subsection{Velocity field inside impacting droplet on heated wall}

As a velocity difference occur within the droplet, we calculated the average velocities in $z$-direction separately for the center and edge parts of the droplet. Figure 5a shows the calculation domains for the center and edge of the droplet. The average velocities of both the center and edge of the droplet were calculated from the six shells of the control volumes. To calculate the center of the droplet, Shell 1 was excluded because the number of molecules in Shell 1 was low for averaging owing to its small volume. The average velocities of the edge were calculated with six shells from the outermost shell. As the droplet formed the adsorption layer at Layer 1 of the control volumes, we also excluded Layer 1 for the calculation of both the center and edge of the droplet.

The time evolutions of the average velocities in $z$-direction of the edge, center, and whole of the droplet $(\alpha=0.14)$ are shown in Fig. $5 \mathrm{~b}$ for $T_{w}=213 \mathrm{~K}$, Fig. $5 \mathrm{c}$ for $T_{w}=255 \mathrm{~K}$, and Fig. $5 \mathrm{~d}$ for $T_{w}=340 \mathrm{~K}$. The circles in Figs. $5 \mathrm{c}$ and $5 \mathrm{~d}$ represent the time when the Leidenfrost effect occurred. Owing to the droplet shape at the instance of impacting and in the spreading process, the average velocities at the edge of the droplet increased faster than at the center of the droplet after impacting (approximately $t<70 \mathrm{ps)}$. For $T_{w}=213 \mathrm{~K}$, little difference was shown in the average velocities within the droplet. Meanwhile, the average velocities at the edge exceeded those of the center and whole of the droplet for $T_{w}=255 \mathrm{~K}$ and $T_{w}=340 \mathrm{~K}$, as shown in Figs. $5 \mathrm{c}$ and $5 \mathrm{~d}$. A higher velocity at the edge of the droplet contributed to the levitation of the entire droplet when $T_{w}=255 \mathrm{~K}$ and $T_{w}=340 \mathrm{~K}$.

After the occurrence of the Leidenfrost effect, the average velocities of the three regions become a same velocity at approximately $V=35 \mathrm{~m} / \mathrm{s}$ for both cases as shown in Figs. 5c and 5d. In general, we can consider that the levitation velocity of a droplet becomes faster as the wall temperature increases. However, the levitation velocities were almost the same for $T_{w}=255 \mathrm{~K}$ and $T_{w}=340 \mathrm{~K}$. When the wall temperature was higher, it took a shorter time to levitate and total evaporation amount became smaller. Our previous MD study indicated that the droplet molecules maintains larger number as the wall temperature was higher [25]. Considering these results, the levitating droplet maintained a larger volume, and the levitation velocity does not become higher when $T_{w}=340 \mathrm{~K}$ in comparison with when $T_{w}=255 \mathrm{~K}$.

We subsequently conducted a more detailed investigation on the velocity inside the impacting droplet to elucidate why the large upward velocity was caused at the edge of the droplet. Figure 6 shows the binarized density fields and the corresponding velocity fields in the $z$ - and $r$-directions at $T_{w}=340 \mathrm{~K}$ for $\alpha=0.14$. The velocity fields in $z$-direction, corresponding to Fig. 5d, are shown in Fig. 6b. As the droplet was assigned the initial velocity toward the solid wall, the droplet has negative velocities in the initial stage of impacting. After impacting, the upward velocity increases significantly at the edge of the droplet in the levitation process, as discussed in the results in Fig. 5d.

Figure $6 \mathrm{c}$ shows the velocity fields in the $r$-direction, which is defined as positive in the radially outward direction. Because the droplet spreads after impacting, the bottom of the droplet indicates a high outward velocity at $t=50 \mathrm{ps}$. After the spreading process, a radial velocity was occurred at the bottom of the droplet owing to heat reception. The dotted green line in Fig. $6 \mathrm{c}$ separates the droplet into upper and lower parts. An outward velocity occurs primarily in the lower part of the droplet while an inward velocity occurs primarily 
(a)

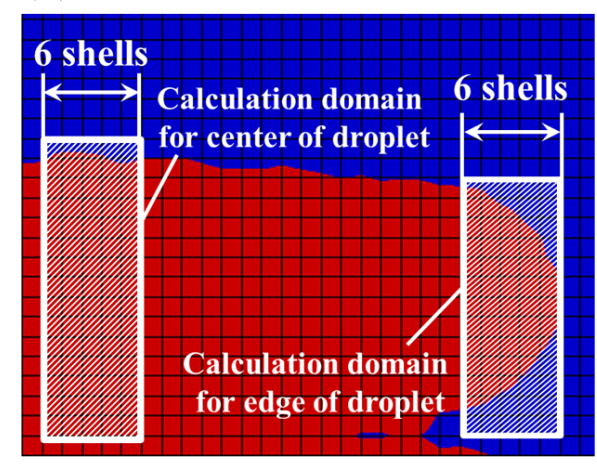

(c) $T_{w}=255 \mathrm{~K}$

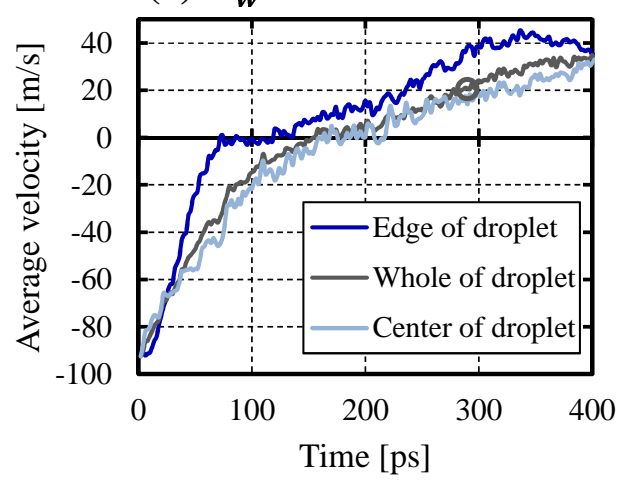

(b) $T_{w}=213 \mathrm{~K}$

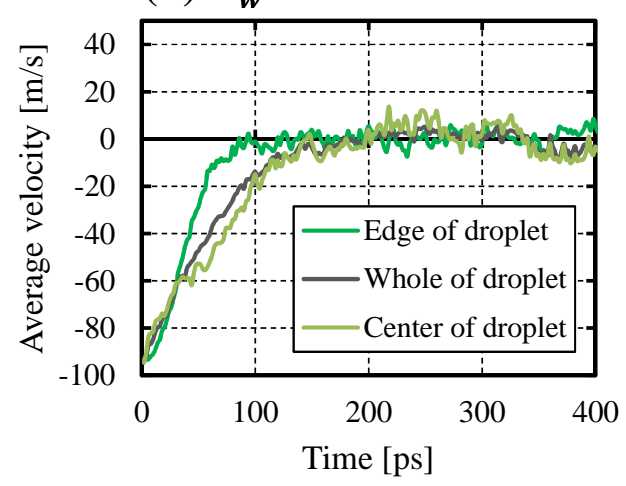

(d) $T_{w}=340 \mathrm{~K}$

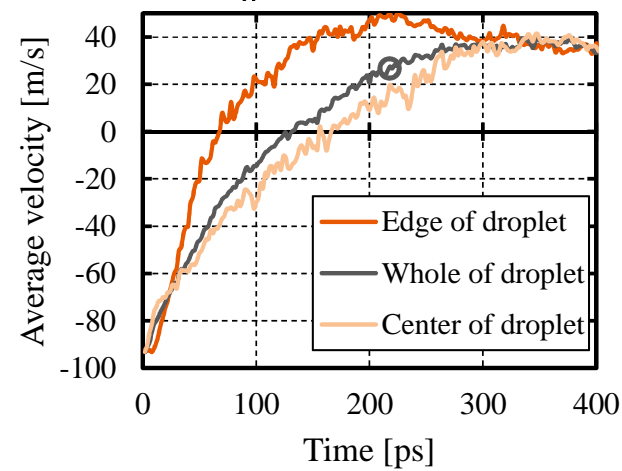

Fig. 5. (a) Schematic of calculation domains for average velocities in $z$-direction of edge and center of droplet. Time evolutions of average velocity of edge, whole, and center of droplet for $\alpha=0.14$ at (b) $T_{w}=213 \mathrm{~K}$, (c) $T_{w}=255 \mathrm{~K}$, and (d) $T_{w}=340 \mathrm{~K}$. Circles in (c) and (d) represent the time Leidenfrost effect occurred.

in the upper part. By comparing and associating with the velocity fields in the $z$-direction, we confirm that an internal flow as that shown in Fig. 7a is generated. The velocity vectors plot on the binalized density field which represents $t=130 \mathrm{ps}$ in Fig. 6a-c is shown in Fig. 7b. We can confirm the generated internal flow also from Fig. 7b. After the Leidenfrost effect occurred $(t=218 \mathrm{ps})$, the $r$-directional velocity field exhibits an inward velocity in the entire droplet because the levitating droplet attempts to return to the spherical shape by its surface tension.

In conclusion, the upward velocity at the edge of the droplet was generated owing to the internal flow, and the impacting droplet can levitate from its edge, which had a higher upward velocity. Because the upward velocity at the edge of the droplet varied with the wall temperature, the relation between the wall temperature and internal flow should be investigated.

\subsection{Temperature field inside impacting droplet on heated wall}

To investigate the influence of the wall temperature on the impacting droplet, we calculated the temperature in a control volume using the following equation:

$$
T=\frac{2}{3 N k_{\mathrm{B}}} \sum_{i}^{N} \frac{1}{2} m_{\mathrm{Ar}}\left(v_{\mathrm{th}}^{i}\right)^{2}
$$

where $N$ is the number of argon molecules in a control volume, $k_{\mathrm{B}}$ is the Boltzmann constant, $m_{\mathrm{Ar}}$ is the mass of the argon molecule, and $v_{\mathrm{th}}^{i}$ is the thermal velocity of the molecule $i$. The thermal velocity is defined as 


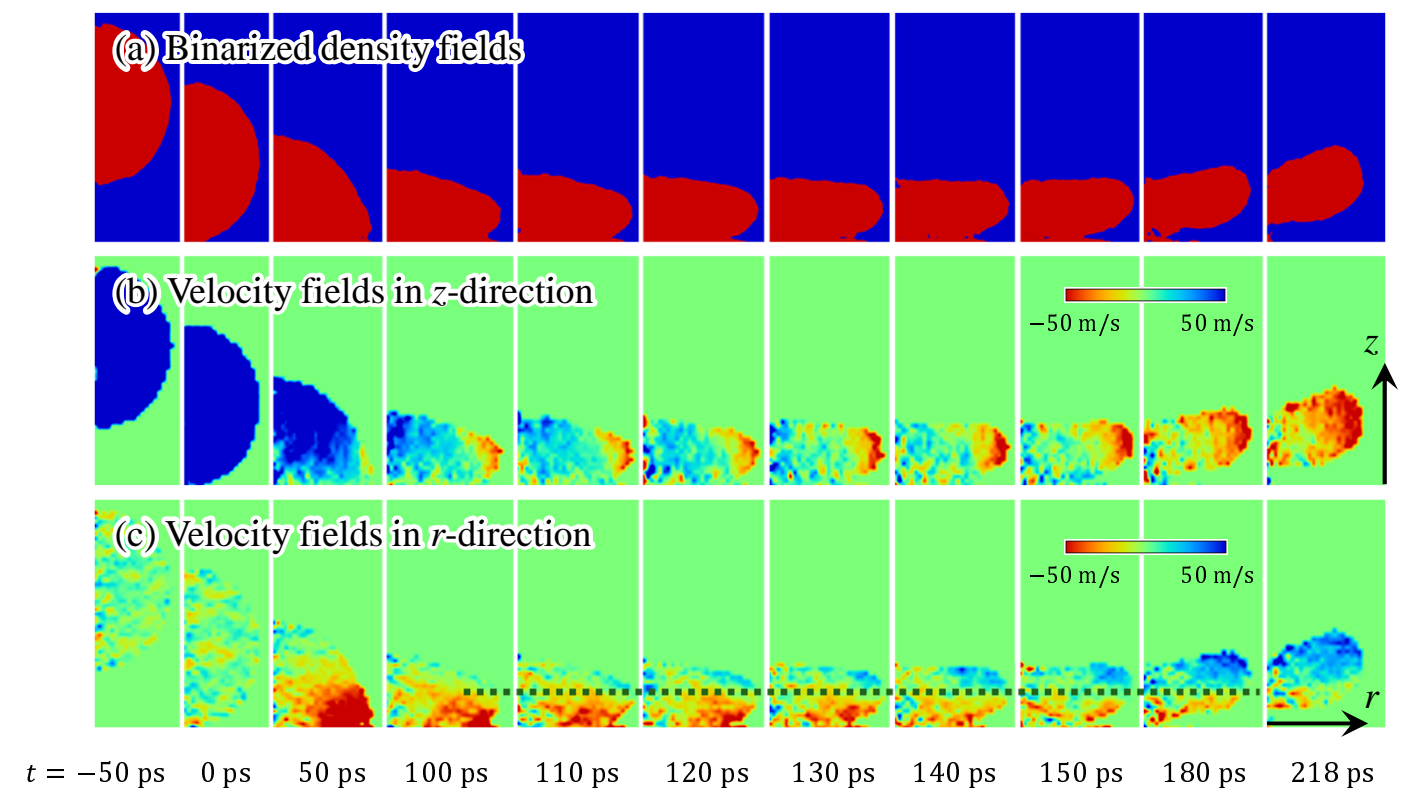

Fig. 6. (a) Binarized density fields of droplet for $\alpha=0.14$ at $T_{w}=340 \mathrm{~K}$. Corresponding velocity fields in (b) $z$-direction and (c) $r$-direction. Dotted green line shown in (c) divides droplet into upper and lower parts. Outward velocity occurred at lower part and inward velocity occurred at upper part.
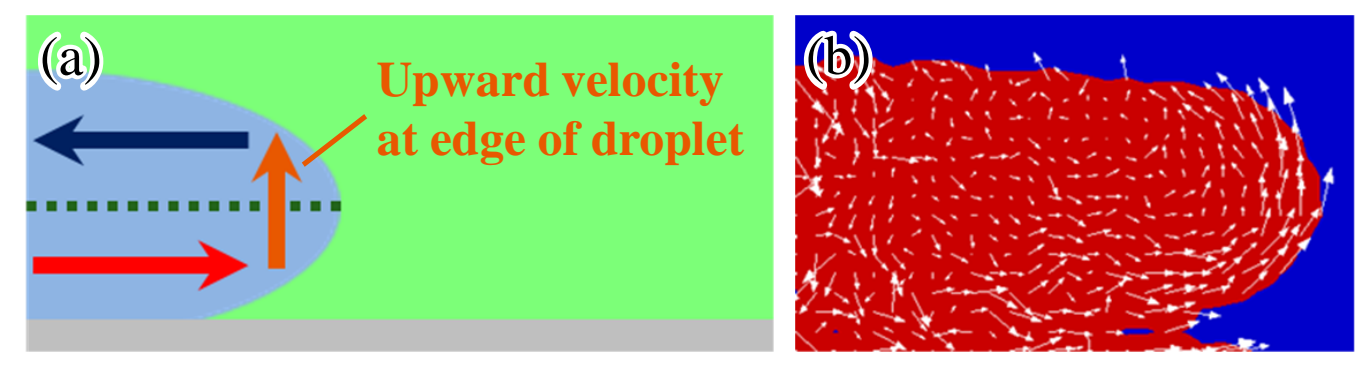

Fig. 7. (a) Schematic of internal flow and caused upward velocity; (b) velocity vectors plot for $\alpha=0.14$ at $T_{w}=340 \mathrm{~K}(t=130 \mathrm{ps})$.

the difference between the velocity of a molecule and average velocity of molecules in a cell of the control volume. The average velocity of each cell of the control volumes and the thermal velocity of molecules were calculated every time step, and the temperature $T$ in Eq. 6 was also calculated every time step. The temperature fields were obtained every 2 ps from the average temperatures for 400 steps in each cell.

Binarized density fields and the corresponding temperature fields of the droplets after impacting are shown in Fig. 8. The temperature of the liquid phase is limited to the critical temperature $T_{\mathrm{c}}$, and the critical temperature which is derived from the Lennard-Jones fluid of argon is $T_{\mathrm{c}}=154.5 \mathrm{~K}$ [33]. Thus, we set the maximum temperature of the temperature fields at $180 \mathrm{~K}$ with a margin. Further, we set the minimum temperature at $80 \mathrm{~K}$ because the initial temperature of the droplet was $T_{0}=85 \mathrm{~K}$. In order to investigate the droplet temperature before the Leidenfrost effect occurred, Fig. $8 \mathrm{~b}$ and $8 \mathrm{c}$ show the temperature fields until the droplet detached from the heated wall. Figure 8 shows that the droplet temperatures increase in the vicinity of the heated wall for all cases. High-temperature regions at the bottom of the droplet become thicker as the wall temperature becomes higher. As shown in Figs. 8b and 8c, the high-temperature regions of the droplet evaporate and disappear before droplet levitation.

Because the droplet separates from Layers 2-4, as shown in Fig. 4, the droplet temperature in Layers 2-4 may affect the detach and evaporation processes of the droplet. The time evolutions of the average 


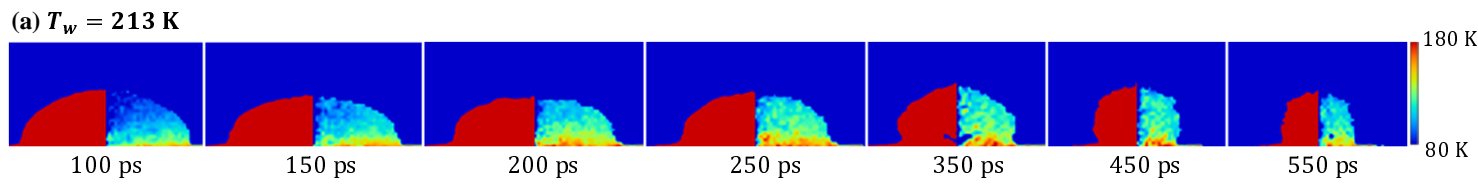

(b) $T_{w}=255 \mathrm{~K}$

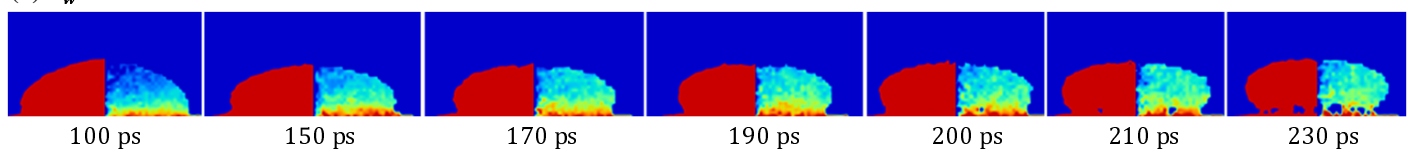

(c) $T_{w}=340 \mathrm{~K}$

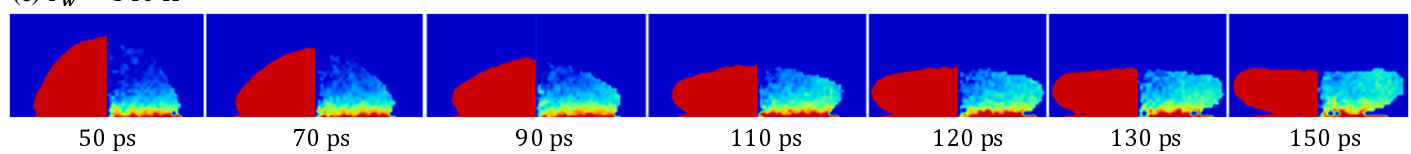

Fig. 8. Binarized density fields (left side) and corresponding temperature fields (right side) for $\alpha=0.14$ at $T_{w}=$ (a) 213, (b) 255 , and (c) $340 \mathrm{~K}$.

temperatures in Layers 2-4 of the droplet $\left(\rho>430 \mathrm{~kg} / \mathrm{m}^{3}\right)$ for each wall temperature are shown in Fig. 9. The dashed red line in Fig. 9 represents the critical temperature of argon $\left(T_{\mathrm{c}}=154.5 \mathrm{~K}\right)$. This figure shows that the average temperatures reach the critical temperature when $T_{w}=255 \mathrm{~K}$ and $340 \mathrm{~K}$. It is reported that heterogeneous nucleations seldom occur on hydrophilic surfaces in nanoscale MD simulations [19, 20]. Thus, the temperatures of the liquid phase exceeded the boiling point and reached the critical temperature locally in the vicinity of the heated wall in this MD study.

The average temperatures in Layers 2-4 reached the critical temperature around $t=200 \mathrm{ps}$ and $120 \mathrm{ps}$ for $T_{w}=255 \mathrm{~K}$ and $340 \mathrm{~K}$, respectively. As shown in Figs. $8 \mathrm{~b}$ and $8 \mathrm{c}$, the droplets in the vicinity of the heated wall gradually starts to change into the vapor phase after these times. This result indicates that the temperature increase of the droplet in the vicinity of the heated wall affects the initial stage of the levitation of the droplet.

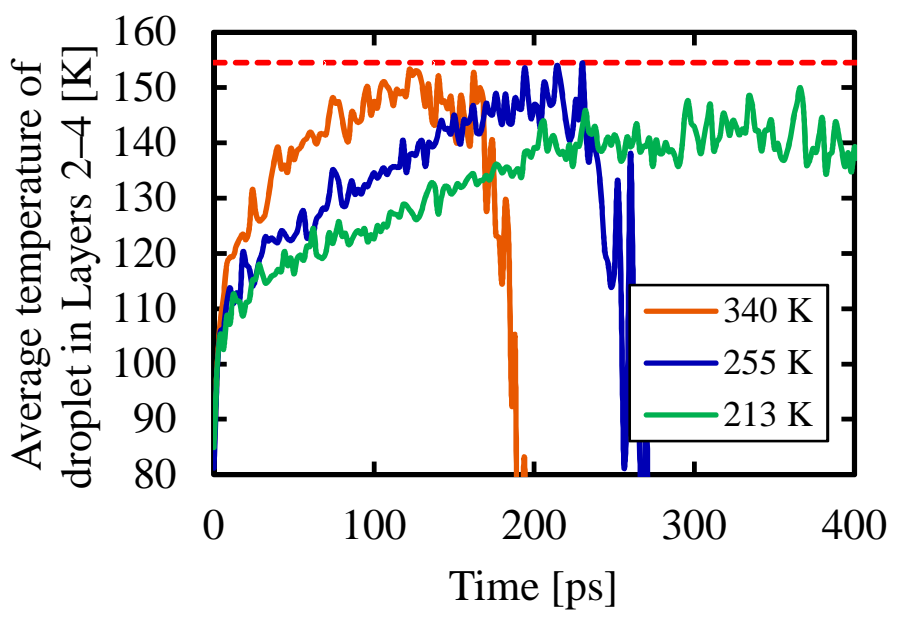

Fig. 9. Time evolutions of average temperature of droplet in Layers 2-4 for $\alpha=0.14$. Dashed red line represents critical temperature of $\operatorname{argon}\left(T_{\mathrm{c}}=154.5 \mathrm{~K}\right)$. 


\subsection{Droplet evaporation on heated wall}

To elucidate the details of the evaporation processes accompanying temperature increase, we calculated the evaporation amounts from the droplet. We defined the evaporation molecules from Layer $i$ as the molecules that were in the liquid phase $\left(\rho>430 \mathrm{~kg} / \mathrm{m}^{3}\right)$ of Layer $i$ at one time and in the vapor phase $\left(\rho \leq 430 \mathrm{~kg} / \mathrm{m}^{3}\right)$ at the following time (after $\left.2 \mathrm{ps}\right)$. Further, we defined the condensation molecules in Layer $i$ as the molecules that were in the vapor phase at one time and in the liquid phase of Layer $i$ at the following time. The difference between the numbers of evaporation and condensation molecules represents the number of net evaporation molecules. The evaporation amount from the droplet was estimated from the number of net evaporation molecules. When a droplet impacts on an unheated wall, liquid molecules are released from the droplet owing to the effect of the impacting. Thus, by this definition, evaporation molecules contain molecules that are released by impacting.

The time evolutions of the total number of net evaporation molecules from each layers of the impacting droplet for $\alpha=0.14$ are shown in Fig. 10a for $T_{w}=85 \mathrm{~K}$ and Fig. $10 \mathrm{~b}$ for $T_{w}=255 \mathrm{~K}$. When $T_{w}=85 \mathrm{~K}$, evaporation by heat reception did not occur and molecules were released only by impacting, as mentioned above. Because the adsorption layer was formed in Layer 1 and the droplet was separated from Layers 2-4 (shown in Fig. 4), we calculated the sum of the number of net evaporation molecules for every three layers except Layer 1. Figures 10a and 10b show that a larger difference occurred in the evaporation amount from Layers 2-4. This implies that the most evaporation occurred at the position close to the heated wall and that heat reception affected little at the regions above. It is widely acknowledged that the evaporation of liquids on a solid wall occurs primarily in the vicinity of the three-phase contact line (TPCL) [36]. Furthermore, a previous research reported that the evaporation amount of a sessile droplet increased significantly on a substrate where the TPCL was increased by nanorods [37]. It means that the TPCL affected the evaporating phenomenon of droplets on the solid wall significantly. As mentioned in Section 3.1, it was reported that the non-evaporating region is present in the vicinity of the contact line $[34,35]$ and this region corresponds to the adsorption layer in Layer 1 of the present study. Moreover, Zhang et al. demonstrated that the evaporation of a droplet occurred preferentially in the TPCL region in nanoscale MD simulation with a hydrophilic surface [16]. In this study, we assumed that the TPCL region is in the vicinity of the contact line in Layers 2-4 from the result of Fig. 10; therefore, we can conclude that it is reasonable to utilize the evaporation amount from Layers 2-4 for the investigations into the droplet evaporation on the heated wall for $\alpha=0.14$.

(a) $T_{w}=85 \mathrm{~K}$

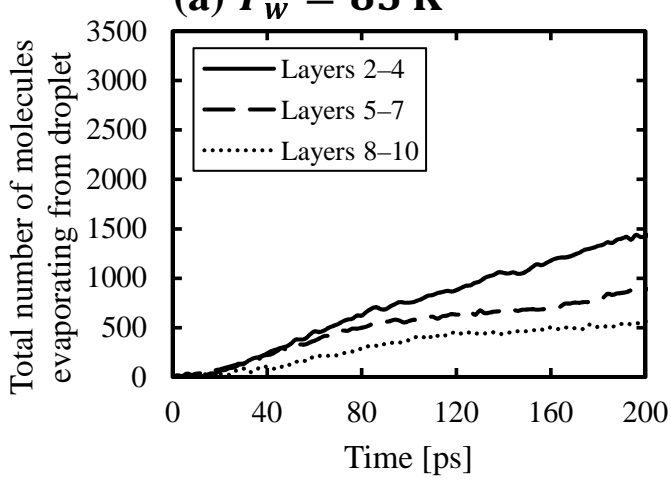

(b) $T_{w}=255 \mathrm{~K}$

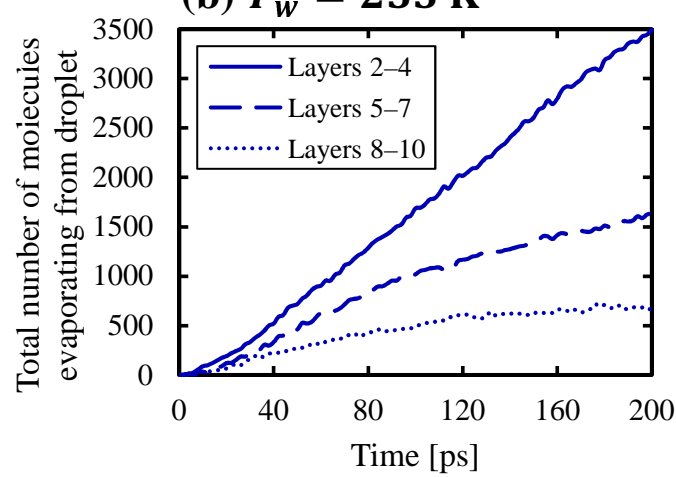

Fig. 10. Time evolutions of total number of net evaporation molecules from each layers for $\alpha=0.14$ at (a) $T_{w}=85 \mathrm{~K}$, and (b) $T_{w}=255 \mathrm{~K}$

\subsection{Evaporation near TPCL and induced radial flow}

Figure 11 shows the time evolutions of the total number of net evaporation molecules from Layers 2-4 for each wall temperature. The circles represent the time when the Leidenfrost effect occurred. Because 
the droplet detached and levitated from Layers 2-4, liquid molecules were almost not present in Layers 2-4 before levitation. Thus, before the Leidenfrost effect occurs, the slopes of the graphs in Fig. 11 become almost flat for $T_{w}=255 \mathrm{~K}$ and $340 \mathrm{~K}$ and it means that the total number of net evaporation molecules does not increase (evaporations does not occur from Layers 2-4).

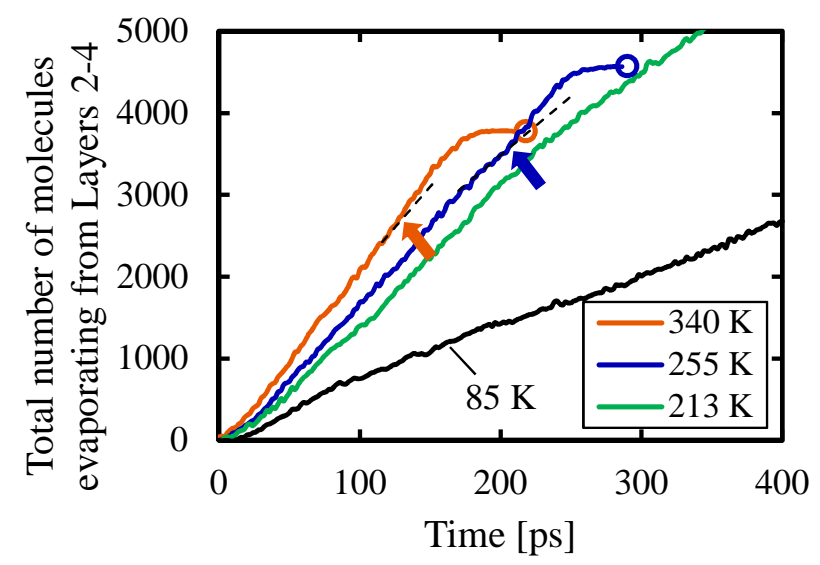

Fig. 11. Time evolutions of total number of net evaporating molecules $(\alpha=0.14)$ from Layers $2-4$ for each wall temperature. Circles represent the time when Leidenfrost effect occurred. Sharp increases occurred at points indicated by solid arrows for $T_{w}=255$ and 340 K. Dashed lines are approximate curves up to these points.

In addition, we confirm that significant increases occurred in the total number of the net evaporation molecules at the points indicated by solid arrows for $T_{w}=255 \mathrm{~K}$ and $340 \mathrm{~K}$ in Fig. 11 . The increases in the slopes imply that the evaporation amount from Layers 2-4 increases temporarily. The significant increases occur at approximately $t=200 \mathrm{ps}$ for $T_{w}=255 \mathrm{~K}$ and $t=120 \mathrm{ps}$ for $T_{w}=340 \mathrm{~K}$. These increases occur when the average temperatures in Layers $2-4$ reach the critical temperature, as shown in Fig. 9. When the droplet temperature in Layers $2-4$ reached the critical temperature, the droplet in this region could not be in the liquid phase and had to change into the vapor phase because the liquid density decreased below $\rho_{\mathrm{c}}$. As a result, the evaporation amount increased significantly and the intensive vapor flow occurred.

Figure 12 shows the density fields and intended positions of the TPCL regions of the impacting droplet at $T_{w}=255 \mathrm{~K}$ in this MD study. The circles on the binarized density fields in the left column of Fig. 12 show the intended positions of the TPCL regions. As a phenomenon related to the droplet evaporation on the solid wall, it was reported that a radial flow at the bottom of the droplet was induced when evaporation occurred in the vicinity of the TPCL $[38,39]$. It was also reported that the TPCL was pinned in 90-95\% of the evaporation process and receded only in the final stage of the evaporation process [40]. Moreover, Gao et al. reported that a contact line was pinned for a longer time on a hydrophilic surface than on a hydrophobic surface [41]. The radial flow is mainly induced while the contact line is pinned. As shown in Fig. 12, the position of the TPCL region moves slightly during evaporation; however, it does not recede significantly when the droplet detached from the heated wall. Thus, in the present MD simulations, although the TPCL region is not pinned perfectly, we assumed that the droplet evaporation from the TPCL region induces a radial flow at the bottom of the droplet. The white rectangle at $t=250 \mathrm{ps}$ in Fig. 12 shows the region in the vicinity of the heated wall after most of the droplet detached from the wall. In the binarized density fields, it appears that the droplet is mostly separated from the wall. However, in the continuous density fields, a vapor-liquid boundary surrounds the liquid phase and there is the vapor film beneath the liquid droplet. Hence, although the liquid phase was not in contact with the heated wall, the heated wall continued to have some effect on the behavior of the liquid phase through the vapor flow.

Figure 13 shows the time evolutions of the average radial velocities in Layers 5-7 for each wall temper- 

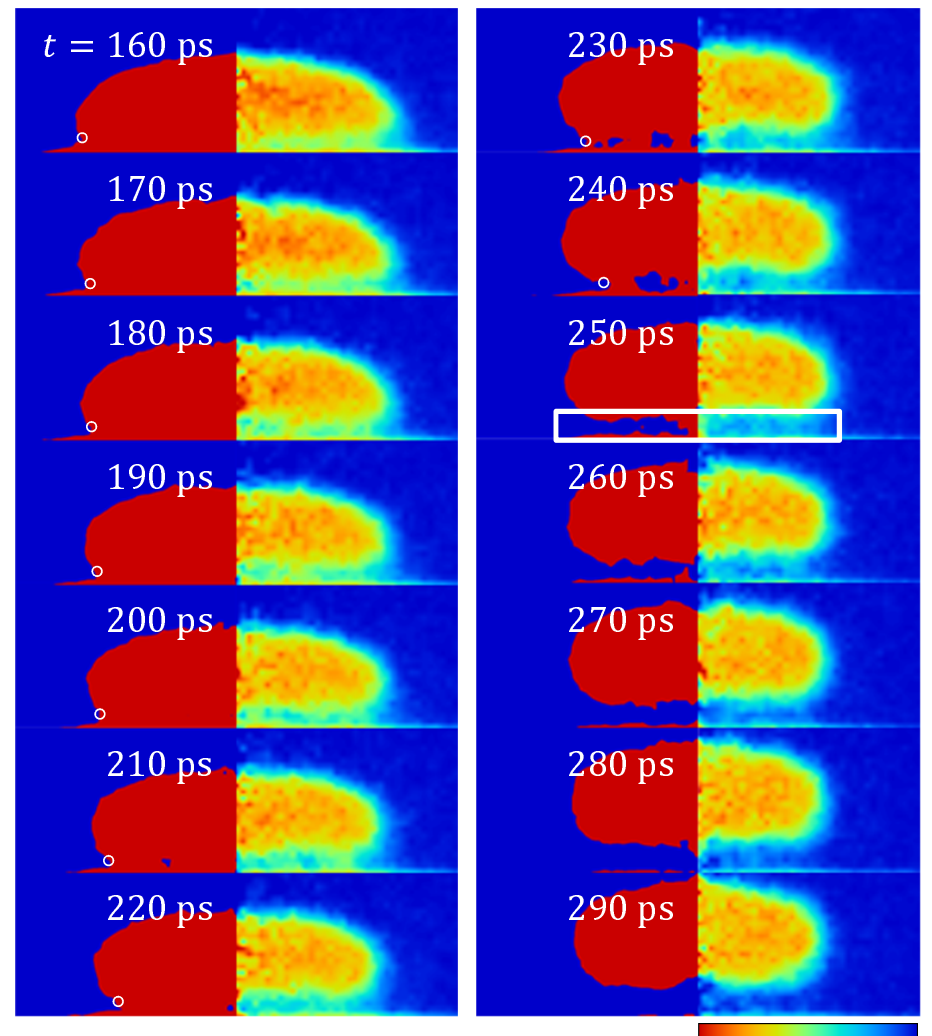

$0 \mathrm{~kg} / \mathrm{m}^{3} \quad 1400 \mathrm{~kg} / \mathrm{m}^{3}$

Fig. 12. Binarized density fields (left side) and continuous density field (right side) of impacting droplet at $T_{w}=255 \mathrm{~K}$ for $\alpha=0.14$. Circles on binarized density fields in left column represent intended positions of three-phase contact line (TPCL).

ature. Because the droplet separates from Layers $2-4$, this region does not maintain the liquid phase during evaporation and levitation processes. Hence, to represent the radial velocities occurring at the bottom of the liquid droplet, we calculated the average radial velocities in Layers 5-7. The dashed lines in Fig. 13 represent the time when the Leidenfrost effect occurred. The average velocity when $T_{w}=85 \mathrm{~K}$, which was not affected by heat reception, is also shown in Fig. 13. It represents only the effect of the impacting. Thus, the difference from the case $T_{w}=85 \mathrm{~K}$ is the velocity that was induced by heat reception. At the early stage of the impacting $(t<50 \mathrm{ps})$, the average radial velocities indicate maximum values for all wall temperatures. This was caused by the droplet spreading owing to the effect of the impacting. Subsequently, the radial velocities decrease owing to viscous dissipation [42] for all cases. When $T_{w}=213 \mathrm{~K}$, the effect of heat reception on the radial outward direction was shown in the initial spreading process $(t<150 \mathrm{ps})$. After spreading $(t>200 \mathrm{ps})$, the average radial velocity at the bottom of the droplet oscillates negative and positive. Eventually, the velocities become approximately $V=4 \mathrm{~m} / \mathrm{s}$ for both cases of $T_{w}=85 \mathrm{~K}$ and $213 \mathrm{~K}$. When $T_{w}=255 \mathrm{~K}$ and $340 \mathrm{~K}$, the average radial velocities increased significantly before the Leidenfrost effect occurred, and the velocities indicate positive values continuously. These increases occurred approximately at $t=200 \mathrm{ps}$ for $T_{w}=255 \mathrm{~K}$ and $t=120 \mathrm{ps}$ for $340 \mathrm{~K}$. These times correspond well to the times when the temperatures of the droplet reached the critical temperature of argon (shown in Fig. 8), and the times when the evaporation amount increased significantly (shown in Fig. 11). This implies that the radial outward velocities were induced significantly by the evaporation on the high-temperature wall. The high wall temperature resulted in a significant increase in the droplet temperature in the vicinity of the heated 
wall, and an intensive evaporation occurred when the droplet temperature reached to the critical temperature of argon. This intensive evaporation from the TPCL region induced a high radial flow at the bottom of the droplet.

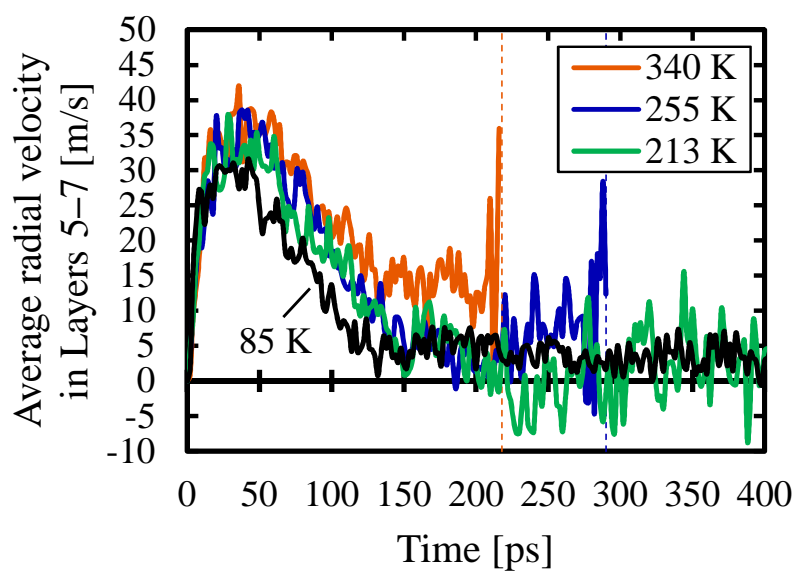

Fig. 13. Time evolutions of average radial flow in Layers 5-7. Dashed lines represent the time when Leidenfrost effect occurred. Significant increases occurred before Leidenfrost effect occurred.

In particular, for $T_{w}=255 \mathrm{~K}$, the upward velocity at the edge of the droplet indicated a significant increase at approximately $t=200 \mathrm{ps}$, as shown in Fig. 5c. This implies that the increase in the radial velocity at the bottom of the droplet and the increase in the upward velocity at the edge of the droplet occurred almost simultaneously. Thus, we can conclude that the high upward velocity at the edge was caused by the internal flow shown in Fig. 7, and this internal flow was caused by the intensive evaporation when the droplet temperature reached the critical temperature. Finally, the impacting droplet levitated from its edge because of the resulted high upward velocity. The series of these phenomena from temperature increase is the mechanism of the Leidenfrost effect in the present MD study.

\subsection{Influence of liquid-solid intermolecular force}

We demonstrated the mechanism of the Leidenfrost effect by varying the wall temperature with the fixed value of $\alpha=0.14$. In this section, we demonstrate the influence of the value of $\alpha$-the liquid-solid intermolecular force - on the levitation mechanism with a fixed wall temperature of $T_{w}=213 \mathrm{~K}$. We varied $\alpha=0.07,0.14$, and 1.0 as mentioned in Section 2. Figure 14 shows the binary density images of the impacting droplet on the heated wall at $T_{w}=213 \mathrm{~K}$ for each value of $\alpha$. When $T_{w}=213 \mathrm{~K}$, the Leidenfrost effect occurred only for $\alpha=1.0$ as shown in Fig. 14. This result shows that the Leidenfrost effect will likely occur when the liquid-solid intermolecular force is strong. Moreover, we can confirm that the stronger liquid-solid intermolecular force led the thicker adsorption layer as shown in Figs. 14b and 14c.

The initial stage of the levitation mechanism, which has been shown, is the temperature increase at the bottom of the droplet. Hence, to elucidate the influence of the strength of the liquid-solid intermolecular force on the levitation mechanism, we calculated the droplet temperatures in the vicinity of the heated wall without adsorption layers. Figure 15a shows the time evolutions of the average temperature at the bottom of the droplet for each value of $\alpha$ at $T_{w}=213 \mathrm{~K}$. The dashed red line in Fig. 15a represents the critical temperature of argon. When $\alpha=0.14$, the adsorption layer was formed in Layer 1, as shown in Fig. 4; further, we calculated the average temperature in three layers above this adsorption layer (Layers 2-4), as shown in Fig. 9. When $\alpha=0.07$, the adsorption layer was not formed on the heated wall, and when $\alpha=1.0$, it was formed in Layers 1-2 owing to the strong liquid-solid intermolecular force. Thus, the average temperatures in Fig. 15a were calculated in Layers 1-3 for $\alpha=0.07$, in Layers 2-4 for $\alpha=0.14$, and in Layers 3-5 for $\alpha=1.0$. Figure 15a shows that the average temperature becomes higher as the value 


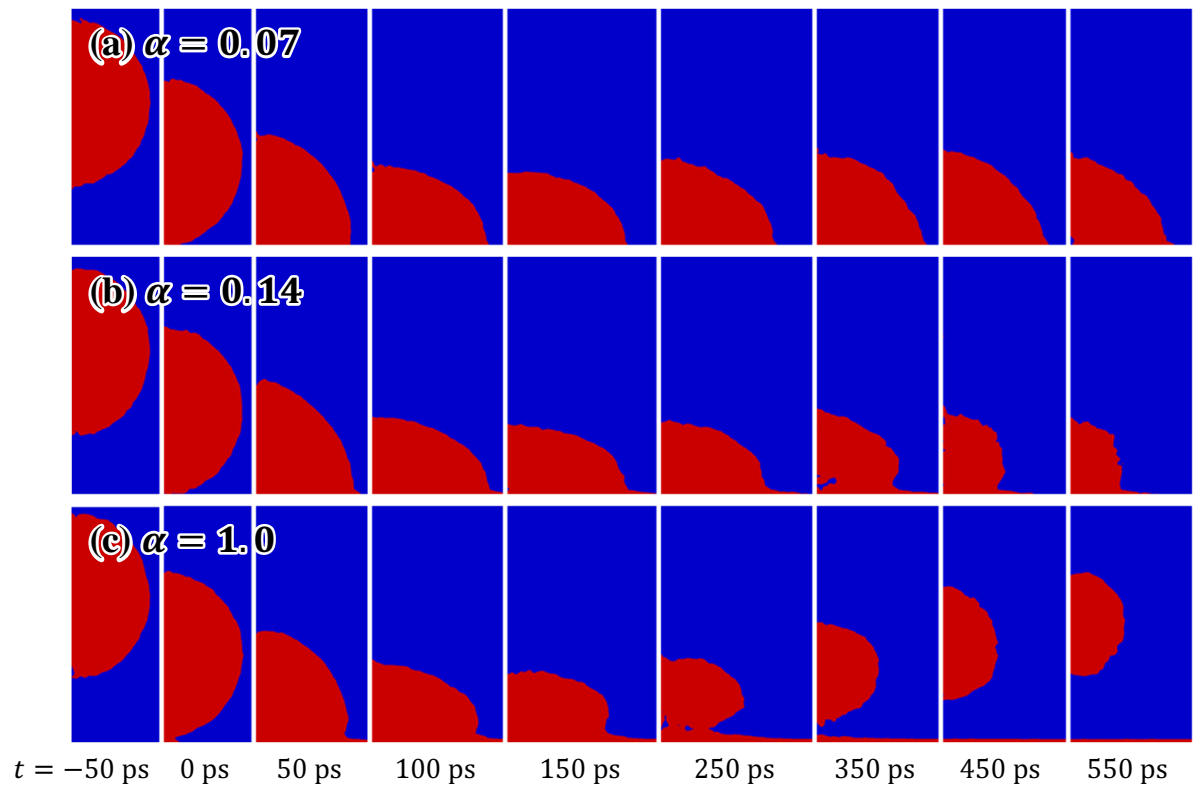

Fig. 14. Binarized density field of impacting droplet on heated wall at $T_{w}=213 \mathrm{~K}$ for (a) $\alpha=0.07$, (b) $\alpha=0.14$, and (c) $\alpha=1.0$.

of $\alpha$ becomes higher, and that the temperature at the bottom of the droplet reaches the critical temperature only when $\alpha=1.0$ at approximately $t=200 \mathrm{ps}$. This implies that the stronger intermolecular force resulted in a significant increase in the droplet temperature although the wall temperature was fixed.

Reaching the critical temperature of the droplet temperature in the vicinity of the heated wall induced the intensive evaporation and significant increase in the radial velocity at the bottom of the droplet, as shown in Figs. 11 and 13. The result of Fig. 15a implies that the same tendencies appear when $\alpha=1.0$. Figure $15 \mathrm{~b}$ shows time evolutions of the total number of net evaporation molecules from the bottom of the droplet. From the calculation domains in Fig. 15a, the evaporation amounts in Fig. 15b were calculated from Layers 1-3 for $\alpha=0.07$, from Layers 2-4 for $\alpha=0.14$, and from Layers 3-5 for $\alpha=1.0$. The open circle in Fig. $15 \mathrm{~b}$ represents the time when the Leidenfrost effect occurred. The evaporation amount increases indicated by the solid arrow for $\alpha=1.0$, and this increase occurred when the droplet temperature reached the critical temperature as shown in Fig. 15a.

A radial flow at the bottom of the droplet on the heated wall is induced even when the value of $\alpha$ is varied. Figure $15 \mathrm{c}$ shows the time evolutions of the average radial velocities at the bottom of the droplet that were induced by the evaporations from the droplet in the vicinity of the heated wall at $T_{w}=213 \mathrm{~K}$ for each value of $\alpha$. The average radial velocities were calculated in Layers 4-6 for $\alpha=0.07$, in Layers 5-7 for $\alpha=0.14$, and in Layers 6-8 for $\alpha=1.0$ (three layers above the evaporating regions) as the regions where the radial velocities were induced. The dashed line represents the time when the Leidenfrost effect occurred. This figure shows that the average radial velocity almost keeps the positive value and shows the steady increase before the Leidenfrost effect occurred when $\alpha=1.0$. The time when this increase occurred is approximately $t=200 \mathrm{ps}$, and corresponds well to the time when the evaporation amount increases in Fig. 15b.

For these results, the temperature increase of the droplet in the vicinity of the heated wall became larger when the liquid-solid intermolecular force was stronger, as well as when the wall temperature was higher; further, the intensive evaporation and induced radial flow at the bottom of the droplet are observed when the droplet temperature reached the critical temperature. As shown in Figs. 5, 6, and 7, the upward velocity was induced by the internal flow, which was caused by the radial flow at the bottom of the droplet. Figure 16 shows the time evolutions of the average velocities of the edge, whole, and center of the droplet for each value of $\alpha$ at $T_{w}=213 \mathrm{~K}$. The calculation domains of each average velocity were the same concept as that 

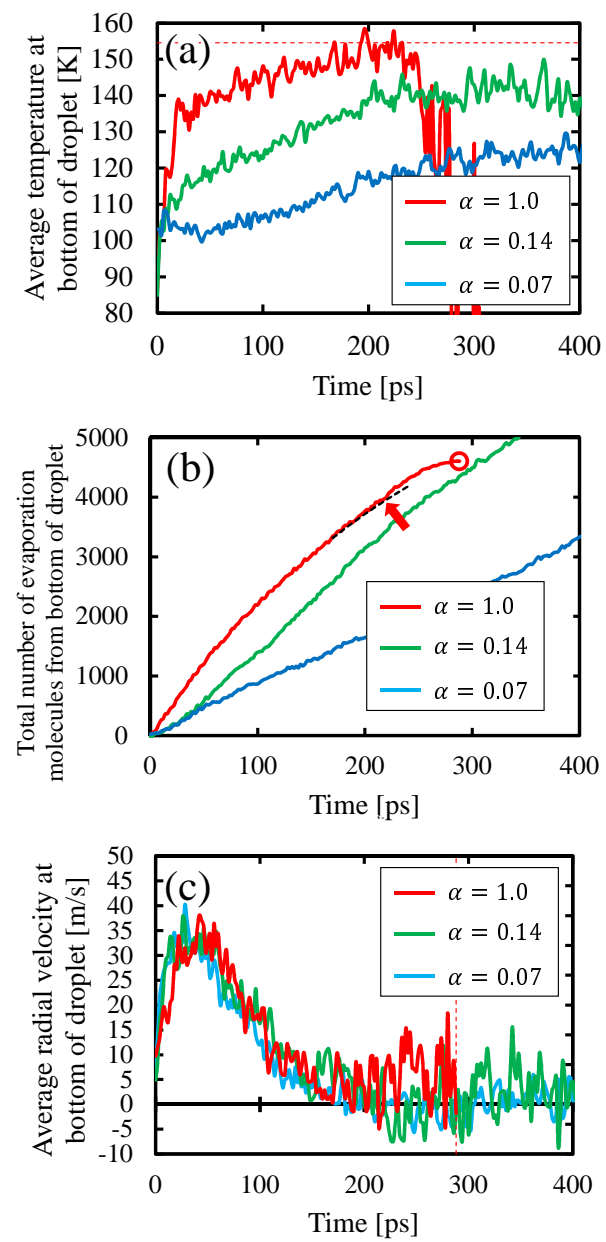

Fig. 15. Time evolutions of (a) average temperature at bottom of droplet, (b) total number of net evaporation molecules from bottom of droplet, and (c) average radial velocity at $T_{w}=213 \mathrm{~K}$. Dashed line in (a) represents critical temperature of argon. Circle in (b) and dashed line in (c) represent the time when Leidenfrost effect occurred.

in Fig. 5a (six shells from Shell 2 and from the outermost shell of the droplet). In Fig. 5, we excluded Layer 1 from the calculation domains because adsorption layers were formed in Layer 1 for $\alpha=0.14$. Thus, for calculating the average velocities in Fig. 16, we excluded Layer 1-2 for $\alpha=1.0$ because the adsorption layers were formed in Layers 1-2, and we did not exclude any layers for $\alpha=0.07$ because adsorption layers were not formed. The open circle in Fig. 16c represents the time when the Leidenfrost effect occurred. When $\alpha=0.07$ and 0.14 , in which the Leidenfrost effect did not occur, the three average velocities indicate little differences after $t=120 \mathrm{ps}$. Meanwhile, for $\alpha=1.0$, the average velocity of the edge of the droplet exceeded those of the others and the Leidenfrost effect occurred, as shown in Fig. 16c. This implies that the upward velocity at the edge of the droplet caused the entire droplet to levitate for $\alpha=1.0$. The times when the radial velocity at the bottom of the droplet increased in Fig. $15 \mathrm{c}$ and when the average velocity at the edge first showed the significant increase in Fig. 16c for $\alpha=1.0$ were both at approximately $t=200$ ps. Hence, the high upward velocity had occurred at the edge of the droplet owing to the internal flow. This levitation mechanism is the same as that of the Leidenfrost effect that occurs when the value of $\alpha$ was constant and the wall temperature became sufficiently high. Thus, we conclude that the liquid-solid intermolecular force primarily affects the temperature increase at the bottom of the droplet, which occurs in the initial stage of 
the levitation mechanism.
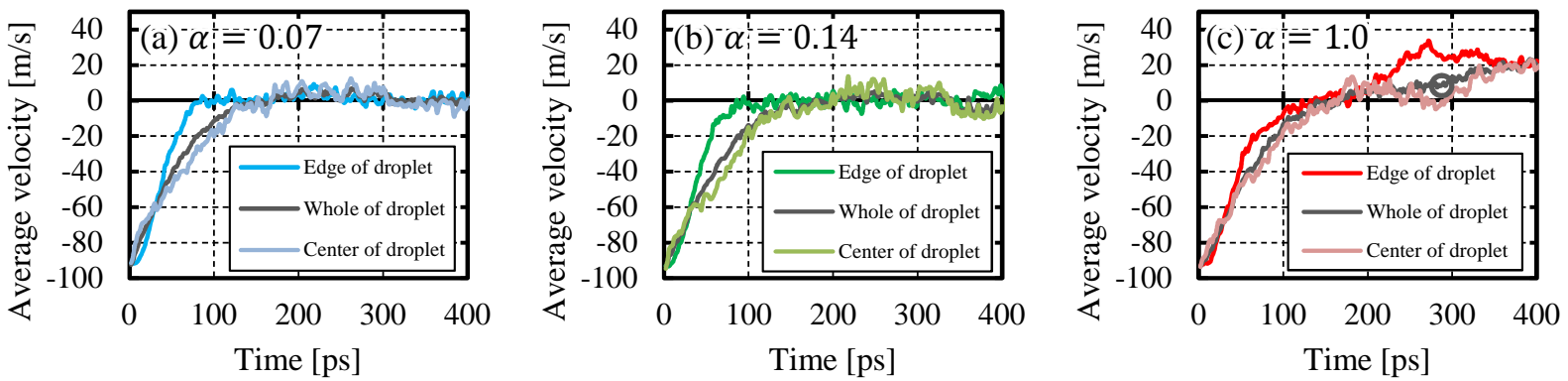

Fig. 16. Time evolutions of average velocity of edge, whole, and center of droplet at $T_{w}=213 \mathrm{~K}$ for (a) $\alpha=0.07$, (b) $\alpha=0.14$, and (c) $\alpha=1.0$. Circle in (c) represents the time when Leidenfrost effect occurred.

\section{Conclusion}

In this study, we conducted MD simulations to clarify the mechanism of the Leidenfrost effect and to elucidate the influence of the liquid-solid intermolecular force on the levitation mechanism for nanodroplets. A high wall temperature or strong liquid-solid intermolecular force results in a significant increase in the nanodroplet temperature in the vicinity of the heated wall. When the temperature reaches the critical temperature, an intensive evaporation occurs and a high radial flow is induced at the bottom of the nanodroplet. This induced radial flow produces the high upward velocity at the edge of the nanodroplet. Consequently, the impacting nanodroplet levitates from its edge. This is the mechanism of the Leidenfrost effect, and the liquid-solid intermolecular force affects the temperature increase at the bottom of the nanodroplet, which occurs in the initial stage of the levitation mechanism.

\section{Reference}

[1] J. G. Leidenfrost, On the fixation of water in diverse fire, International Journal of Heat and Mass Transfer 9 (11) (1966) 11531166.

[2] D. Quéré, Leidenfrost dynamics, Annual Review of Fluid Mechanics 45 (2013) 197-215.

[3] T. Tran, H. J. Staat, A. Prosperetti, C. Sun, D. Lohse, Drop impact on superheated surfaces, Physical review letters 108 (3) (2012) 036101.

[4] W. Zhang, T. Yu, J. Fan, W. Sun, Z. Cao, Droplet impact behavior on heated micro-patterned surfaces, Journal of Applied Physics 119 (11) (2016) 114901.

[5] D. Orejon, K. Sefiane, Y. Takata, Effect of ambient pressure on leidenfrost temperature, Physical Review E 90 (5) (2014) 053012.

[6] M. A. van Limbeek, P. B. Hoefnagels, M. Shirota, C. Sun, D. Lohse, Boiling regimes of impacting drops on a heated substrate under reduced pressure, Physical Review Fluids 3 (5) (2018) 053601.

[7] Y. Takata, S. Hidaka, A. Yamashita, H. Yamamoto, Evaporation of water drop on a plasma-irradiated hydrophilic surface, International journal of heat and fluid flow 25 (2) (2004) 320-328.

[8] I. U. Vakarelski, N. A. Patankar, J. O. Marston, D. Y. Chan, S. T. Thoroddsen, Stabilization of leidenfrost vapour layer by textured superhydrophobic surfaces, Nature 489 (7415) (2012) 274.

[9] C. E. Clavijo, J. Crockett, D. Maynes, Hydrodynamics of droplet impingement on hot surfaces of varying wettability, International Journal of Heat and Mass Transfer 108 (2017) 1714-1726.

[10] D. Arnaldo del Cerro, Á. G. Marín, G. R. Römer, B. Pathiraj, D. Lohse, A. J. Huis in ' t Veld, Leidenfrost point reduction on micropatterned metallic surfaces, Langmuir 28 (42) (2012) 15106-15110.

[11] T. Tran, H. J. Staat, A. Susarrey-Arce, T. C. Foertsch, A. van Houselt, H. J. Gardeniers, A. Prosperetti, D. Lohse, C. Sun, Droplet impact on superheated micro-structured surfaces, Soft Matter 9 (12) (2013) 3272-3282.

[12] H. Kim, B. Truong, J. Buongiorno, L.-W. Hu, On the effect of surface roughness height, wettability, and nanoporosity on leidenfrost phenomena, Applied Physics Letters 98 (8) (2011) 083121.

[13] H. Nair, H. J. Staat, T. Tran, A. van Houselt, A. Prosperetti, D. Lohse, C. Sun, The leidenfrost temperature increase for impacting droplets on carbon-nanofiber surfaces, Soft matter 10 (13) (2014) 2102-2109. 
[14] A. Morshed, T. C. Paul, J. A. Khan, Effect of nanostructures on evaporation and explosive boiling of thin liquid films: a molecular dynamics study, Applied Physics A 105 (2) (2011) 445-451.

[15] J. Yu, H. Wang, A molecular dynamics investigation on evaporation of thin liquid films, International journal of heat and mass transfer 55 (4) (2012) 1218-1225.

[16] J. Zhang, F. Leroy, F. Müller-Plathe, Evaporation of nanodroplets on heated substrates: a molecular dynamics simulation study, Langmuir 29 (31) (2013) 9770-9782.

[17] A. Hens, R. Agarwal, G. Biswas, Nanoscale study of boiling and evaporation in a liquid ar film on a pt heater using molecular dynamics simulation, International Journal of Heat and Mass Transfer 71 (2014) 303-312.

[18] S. Zhang, F. Hao, H. Chen, W. Yuan, Y. Tang, X. Chen, Molecular dynamics simulation on explosive boiling of liquid argon film on copper nanochannels, Applied thermal engineering 113 (2017) 208-214.

[19] G. Nagayama, T. Tsuruta, P. Cheng, Molecular dynamics simulation on bubble formation in a nanochannel, International Journal of Heat and Mass Transfer 49 (23) (2006) 4437-4443.

[20] X. She, T. A. Shedd, B. Lindeman, Y. Yin, X. Zhang, Bubble formation on solid surface with a cavity based on molecular dynamics simulation, International Journal of Heat and Mass Transfer 95 (2016) 278-287.

[21] Y. Liu, X. Zhang, Molecular dynamics simulation of nanobubble nucleation on rough surfaces, The Journal of Chemical Physics 146 (16) (2017) 164704.

[22] S. Gao, Q. Liao, W. Liu, Z. Liu, Effects of solid fraction on droplet wetting and vapor condensation: a molecular dynamic simulation study, Langmuir 33 (43) (2017) 12379-12388.

[23] N. Sedighi, S. Murad, S. K. Aggarwal, Molecular dynamics simulations of nanodroplet spreading on solid surfaces, effect of droplet size, Fluid Dynamics Research 42 (3) (2010) 035501.

[24] D. T. Semiromi, A. Azimian, Molecular dynamics simulation of nonodroplets with the modified lennard-jones potential function, Heat and mass transfer 47 (5) (2011) 579-588.

[25] H. Tabe, K. Kobayashi, H. Yaguchi, H. Fujii, M. Watanabe, Influence of liquid-solid intermolecular force on levitation of impacting nanodroplet, Heat and Mass Transfer 55 (4) (2019) 993-1003.

[26] P. Yi, D. Poulikakos, J. Walther, G. Yadigaroglu, Molecular dynamics simulation of vaporization of an ultra-thin liquid argon layer on a surface, International Journal of Heat and Mass Transfer 45 (10) (2002) 2087-2100.

[27] H. Yaguchi, T. Yano, S. Fujikawa, Molecular dynamics study of vapor-liquid equilibrium state of an argon nanodroplet and its vapor, Journal of Fluid Science and Technology 5 (2) (2010) 180-191.

[28] J. Delhommelle, P. Millié, Inadequacy of the lorentz-berthelot combining rules for accurate predictions of equilibrium properties by molecular simulation, Molecular Physics 99 (8) (2001) 619-625.

[29] X.-D. Din, E. E. Michaelides, Kinetic theory and molecular dynamics simulations of microscopic flows, Physics of Fluids 9 (12) (1997) 3915-3925.

[30] J.-L. Barrat, L. Bocquet, Large slip effect at a nonwetting fluid-solid interface, Physical review letters 82 (23) (1999) 4671.

[31] I. S. Bayer, C. M. Megaridis, Contact angle dynamics in droplets impacting on flat surfaces with different wetting characteristics, Journal of Fluid Mechanics 558 (2006) 415-449.

[32] K. Kobayashi, K. Konno, H. Yaguchi, H. Fujii, T. Sanada, M. Watanabe, Early stage of nanodroplet impact on solid wall, Physics of Fluids 28 (3) (2016) 032002.

[33] D. Heyes, The lennard-jones fluid in the liquid-vapour critical region, Computational Methods in Science and Technology 21 (4) (2015) 169-179.

[34] K. Park, K.-J. Noh, K.-S. Lee, Transport phenomena in the thin-film region of a micro-channel, International Journal of Heat and Mass Transfer 46 (13) (2003) 2381-2388.

[35] H. Wang, S. V. Garimella, J. Y. Murthy, Characteristics of an evaporating thin film in a microchannel, international journal of heat and mass transfer 50 (19-20) (2007) 3933-3942.

[36] P. Stephan, C. Busse, Analysis of the heat transfer coefficient of grooved heat pipe evaporator walls, International Journal of heat and mass transfer 35 (2) (1992) 383-391.

[37] D. P. Singh, J. P. Singh, Enhanced evaporation of sessile water droplet on vertically standing ag nanorods film, The Journal of Physical Chemistry C 115 (24) (2011) 11914-11919.

[38] R. D. Deegan, O. Bakajin, T. F. Dupont, G. Huber, S. R. Nagel, T. A. Witten, Capillary flow as the cause of ring stains from dried liquid drops, Nature 389 (6653) (1997) 827.

[39] R. D. Deegan, O. Bakajin, T. F. Dupont, G. Huber, S. R. Nagel, T. A. Witten, Contact line deposits in an evaporating drop, Physical review E 62 (1) (2000) 756.

[40] H. Hu, R. G. Larson, Evaporation of a sessile droplet on a substrate, The Journal of Physical Chemistry B 106 (6) (2002) 1334-1344.

[41] M. Gao, P. Kong, L.-x. Zhang, Evaporation dynamics of different sizes sessile droplets on hydrophilic and hydrophobic heating surface under constant wall heat fluxes conditions, International Communications in Heat and Mass Transfer 93 (2018) 93-99.

[42] X.-H. Li, X.-X. Zhang, M. Chen, Estimation of viscous dissipation in nanodroplet impact and spreading, Physics of Fluids 27 (5) (2015) 052007. 